\title{
Expedient synthesis of 3-hydroxyisoquinolines and 2-hydroxy-1,4-naphthoquinones via one-pot aryne acyl-alkylation/condensation $\uparrow$
}

\author{
Kevin M. Allan, Boram D. Hong and Brian M. Stoltz* \\ Received 6th July 2009, Accepted 9th September 2009 \\ First published as an Advance Article on the web 6th October 2009 \\ DOI: 10.1039/b913336d
}

A convenient method is disclosed for the synthesis of both 3-hydroxyisoquinolines and

2-hydroxy-1,4-naphthoquinones from $\beta$-ketoesters using a one-pot aryne acyl-alkylation/condensation procedure. When performed in conjunction with a one-step method for the synthesis of the $\beta$-ketoester substrates, this method provides a new route to these polyaromatic structures in only two steps from commercially available carboxylic acid starting materials. The utility of this approach is demonstrated in the synthesis of the atropisomeric P,N-ligand, QUINAP.

Synthetic chemists are constantly in search of new reaction sequences to convert simple, readily available starting materials into increasingly complex products. The most useful and economically valuable of these processes are able to do so quickly and with little operational difficulty. ${ }^{1}$ It was with this concept in mind that we designed a procedure for the conversion of $\beta$-ketoesters to either 3-hydroxyisoquinolines or 2-hydroxy-1,4-naphthoquinones using a novel one-pot aryne acyl-alkylation/condensation sequence. Furthermore, when this process is coupled with a one-step synthesis of the $\beta$-ketoester substrates, it provides an exceptionally general two-step procedure for the conversion of readily available carboxylic acids to either of these important bicyclic aromatic structures. ${ }^{2}$

Traditional approaches to the incorporation of these aromatic functionality within larger molecular scaffolds have typically

The Arnold and Mabel Beckman Laboratories of Chemical Synthesis, Division of Chemistry and Chemical Engineering, California Institute of Technology, 1200 E. California Boulevard, MC 164-30, Pasadena, CA 91125, USA. E-mail: stoltz@caltech.edu; Fax: +1 626564 9297; Tel: +1 6263956064

$\dagger$ Electronic supplementary information (ESI) available: General experimental procedures, characterization data, NMR, and IR spectra. See DOI: $10.1039 / \mathrm{b} 913336 \mathrm{~d}$ relied upon transition metal-catalyzed cross coupling reactions employing $\mathrm{C}\left(\mathrm{sp}^{2}\right)-\mathrm{X}$ precursors. ${ }^{3}$ As an alternative to this strategy, we have been exploring methods to prepare various heteroaromatic systems via condensation of arynes with derivatives of carboxylic acids. ${ }^{4}$ Following our report of a fluoride-induced insertion reaction between $\beta$-ketoesters (1) and arynes derived from silyl aryl triflates (2), ${ }^{5}$ we began to investigate avenues by which the acyl-alkylated arene products (3) could be advanced toward larger ring systems (Scheme 1). ${ }^{6}$ Our interest in nitrogencontaining heterocycles ${ }^{7}$ led us to a report by Bentley et al., in which the synthesis of both 3-hydroxyisoquinolines (4) and 2hydroxy-1,4-naphthoquinones (5) is accomplished by exposure of 1,5-ketoesters similar to 3 to either aqueous ammonia or alkaline base under an ambient atmosphere, respectively. ${ }^{8}$ Indeed, we found that treatment of a crude acyl-alkylation reaction mixture containing methyl (2-acetylphenyl)acetate (3) with aqueous ammonium hydroxide furnished 1-methyl-3-hydroxyisoquinoline (4), ${ }^{9}$ while addition of sodium methoxide generated lawsone (5), an isolate from the henna plant Lawsonia inermis. ${ }^{10}$

Focusing on the first of these two transformations, we investigated the reaction between methyl acetoacetate (1) and a number of functionalized aryne precursors (6) (Table 1). The sequence proved quite general, providing rapid access to a number of

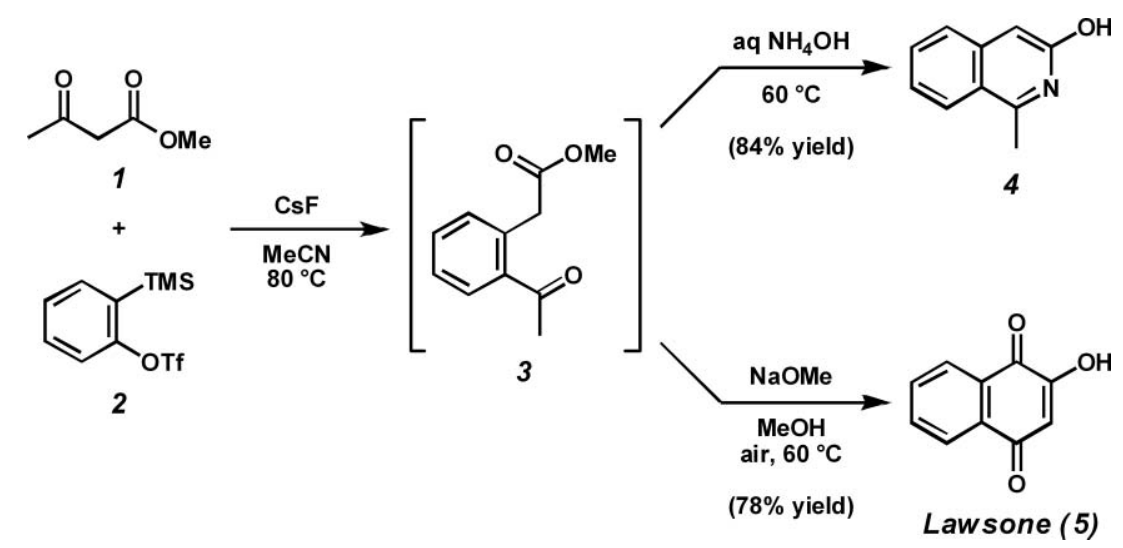

Scheme 1 Aryne acyl-alkylation followed by condensation with ammonia or intramolecular condensation and oxidation. 
Table 1 Synthesis of 3-hydroxyisoquinolines via one-pot aryne acylalkylation and condensation with ammonia

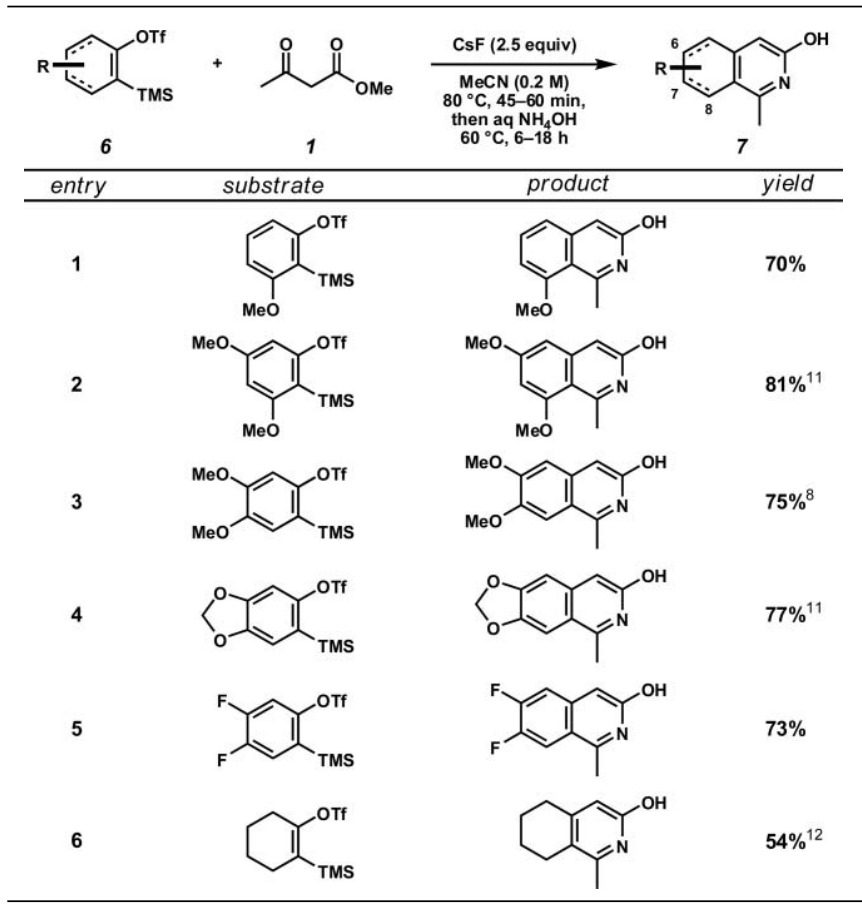

3-hydroxyisoquinoline products (7) featuring substitution at carbons 6,7 , and 8 . The reaction proceeded well with both electron-rich (entries 1-4) and electron-deficient (entry 5) arynes, as well as with cyclohexyne ${ }^{13}$ (entry 6). Notably, the use of unsymmetrically substituted arynes (entries 1 and 2) provided single product isomers originating from regioselective addition of the $\beta$-ketoester to the inductively activated carbon meta to the heteroatom. ${ }^{14}$

Next, we considered alternative $\beta$-ketoesters for the introduction of substitution at $\mathrm{C}(1)$ of the hydroxyisoquinoline scaffold (Table 2). Though methyl acetoacetate and a handful of other $\beta$-ketoesters are commercially available, we found it necessary to prepare additional substrates in order to probe the scope of the reaction. While there are a number of methods to generate $\beta$-ketoesters from the corresponding carboxylic acids (8), we selected a procedure reported by Masamune and coworkers, which employs the addition of a magnesium methylmalonate to a preformed acyl imidazole. ${ }^{15}$ Both simple and high yielding, this method facilitated the synthesis of a small library of differentially substituted methyl $\beta$-ketoesters (9) from readily available materials in a single step. ${ }^{16}$

With a collection of substrates in hand, we set about testing the steric and electronic tolerances of our acyl-alkylation/ condensation reaction sequence. Under optimized conditions, the $\beta$-ketoester (9) and silyl aryl triflate (6) are combined in a Schlenk flask and heated in the presence of CsF. Following formation of the acyl-alkylated arene (e.g., 3), ammonium hydroxide is added and the condensation is allowed to proceed in a sealed environment. We were delighted to find that the reaction proceeds quite well with a variety of alkyl (entries 1 and 2), aryl (entries 3-5), and heteroaryl groups (entries 6-8) located at the $\gamma$-position of the $\beta$-ketoester. In addition, naphthyl isoquinoline (entry 9) and
Table 2 Two-step synthesis of 3-hydroxyisoquinolines from carboxylic acids $^{16}$

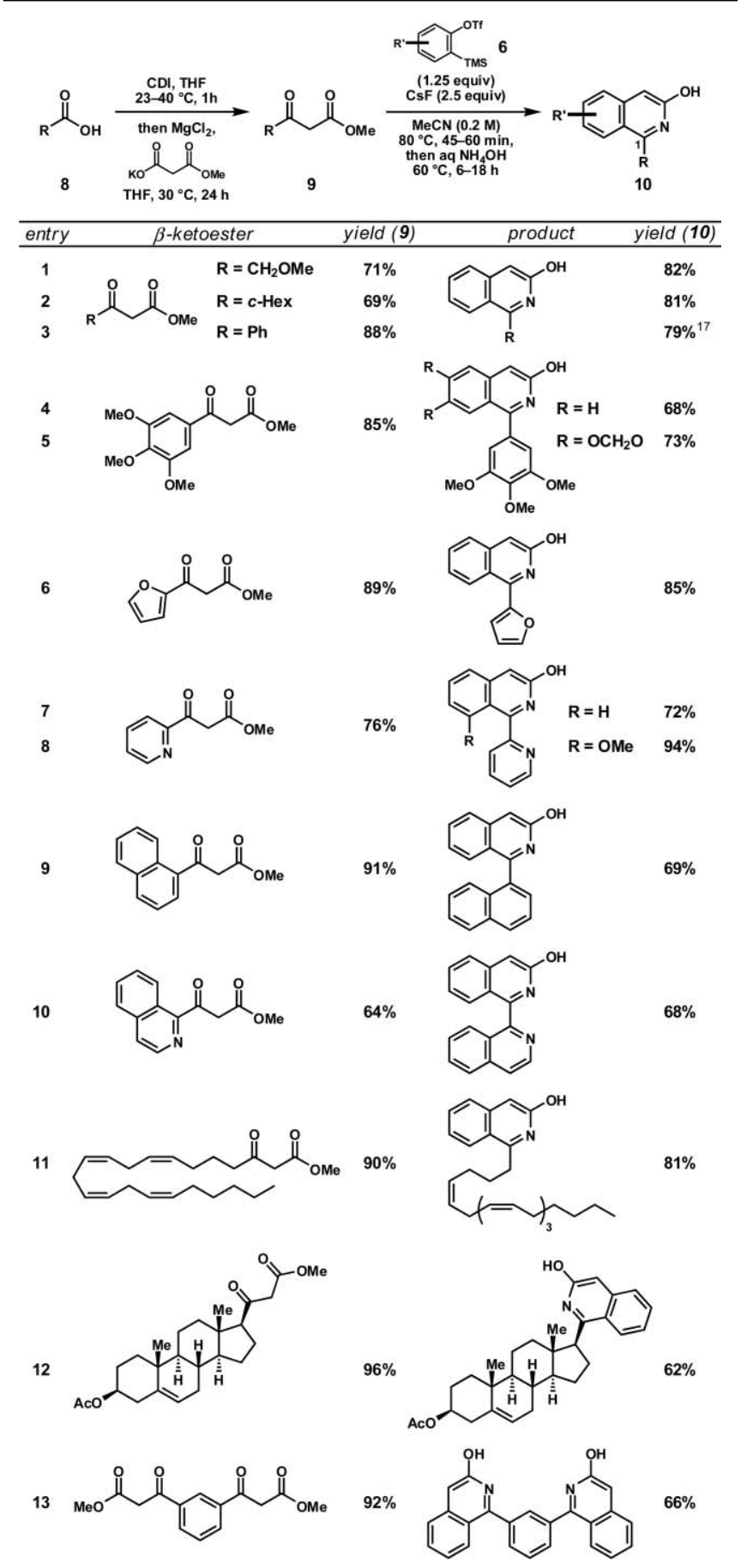

bis-isoquinoline (entry 10) scaffolds - structures central to many ligand frameworks - are accessible. ${ }^{18}$ Interestingly, this sequence proves quite effective at rapidly appending isoquinoline motifs on to biological substrates such as arachidonic acid (entry 11) and $3 \beta$-acetoxy-5-etienic acid (entry 12 ) without epimerization of existing stereocenters. The reaction can even be performed bidirectionally with a diacid to generate a bis(3-hydroxyisoquinoline) product (entry 13). Furthermore, the hydroxyl group provides an excellent functional handle for the introduction of additional substitution 


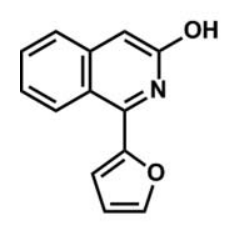

11

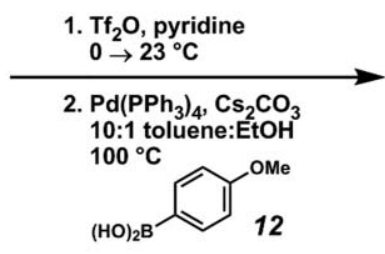

( $85 \%$ yield, 2 steps)<smiles>COc1ccc(-c2cc3ccccc3c(-c3ccco3)n2)cc1</smiles>

13

Scheme 2 Synthesis of a 1,3-diarylisoquinoline.

at $\mathrm{C}(3)$ of the isoquinoline. As a demonstration of this fact, we carried out the triflation of 3-hydroxyisoquinoline 11 (Table 2, entry 6) followed by a palladium-catalyzed Suzuki coupling with 4-methoxyphenylboronic acid (12) to furnish diarylisoquinoline 13 in high yield (Scheme 2). Thus, this method provides avenues not only to $\mathrm{C}(1)$ functionalized hydroxyisoquinolines, but toward 1,3-disubstituted isoquinolines as well.

As previously mentioned, our aryne acyl-alkylation/ condensation reaction sequence enables rapid assembly of structural motifs that are key to several chiral ligand frameworks. In particular, we envisioned that naphthyl isoquinoline 14 (Table 2, entry 9) could be employed toward the synthesis of 1-(2'-diphenylphosphino-1'-naphthyl)-isoquinoline (QUINAP, 18), ${ }^{19}$ an axially chiral $\mathrm{P}, \mathrm{N}$-ligand that has found widespread application in asymmetric catalysis, including enantioselective hydroboration, ${ }^{20}$ allylic alkylation, ${ }^{21}$ and hydrogenation ${ }^{22}$ (Scheme 3). Having developed an expedient method for the construction of naphthyl 3-hydroxyisoquinoline 14, we set out to demonstrate the feasibility of our synthetic approach. Following the acyl-alkylation/condensation sequence, the resulting hydroxyisoquinoline (14) is subjected to triflation. Next, reduction of triflate $\mathbf{1 5}$ yields naphthyl isoquinoline 16, which is subsequently brominated via a nitrogen-directed palladium-catalyzed $\mathrm{C}-\mathrm{H}$ activation. ${ }^{23}$ Finally, a modified version of Buchwald's phosphinearyl bromide coupling ${ }^{24}$ affords the desired target, QUINAP (18). Whereas the majority of previous approaches rely on transition metal-catalyzed cross coupling reactions to form the axial $\mathrm{C}-\mathrm{C}$ bond, ${ }^{25}$ our synthetic route represents a novel disconnection that ultimately builds the isoquinoline ring system on to the existing framework of naphthoic acid.

The second class of compounds, 2-hydroxy-1,4-naphthoquinones, is generated via intramolecular condensation of the acylalkylated arene to form an intermediate 1,3-naphthalenediol, which then autooxidizes under an ambient atmosphere. We first encountered these compounds while working toward 3 -hydroxyisoquinolines when we noticed that $\beta$-ketoester substrates bearing an acidic $\gamma$-methylene (20) preferentially formed the hydroxynaphthoquinone, albeit in low yield, through the action of $\mathrm{NH}_{4} \mathrm{OH}$ as a base. When aqueous potassium carbonate was added in its place and the mixture was heated while open to air, the reaction proceeded cleanly to the hydroxynaphthoquinone (21) (Table 3). ${ }^{26}$ Using these optimized conditions, we are able to generate a variety of hydroxynaphthoquinones possessing alkoxy (entry 1), aryl (entries 2-6), and even indole (entries 7 and 8) substitution at $\mathrm{C}(3)$. However, having optimized the reaction for $\beta$-ketoesters bearing electron-withdrawing functionality at the $\gamma$-position, it was necessary to select a stronger base in order to target alkyl substituted products. Fortunately, sodium methoxide provided a suitable alternative in such cases, giving rise to simple natural product scaffolds such as phthiocol ${ }^{27}$ (entry 9) and $O$-des-methyl-stoechadone ${ }^{28}$ (entry 10 ), as well as a derivative of arachidonic acid (entry 11). Under both conditions, differentially substituted arynes (entries 4, 8, and 10) and cyclohexyne (entry 5) performed comparably to benzyne (derived from $\mathbf{2}$ ).<smiles>Oc1cc2ccccc2c(-c2cccc3ccccc23)n1</smiles>

14

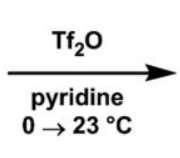

(99\% yield)

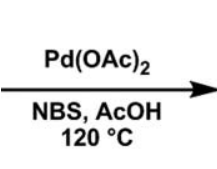

(15\% yield)<smiles>COc1cc2ccccc2c(-c2cccc3ccccc23)n1</smiles>

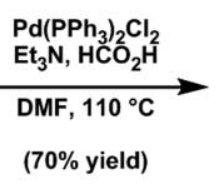<smiles>c1ccc2c(-c3nccc4ccccc34)cccc2c1</smiles>

16<smiles>Brc1ccc2ccccc2c1-c1nccc2ccccc12</smiles>

17

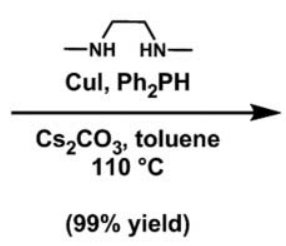

(99\% yield)<smiles>Pc1cccc(-c2ccccc2)c1-c1nccc2ccccc12</smiles>

QUINAP (18)

Scheme 3 Synthesis of QUINAP 
Table 3 Two-step synthesis of 2-hydroxy-1,4-naphthoquinones from carboxylic acids ${ }^{16}$

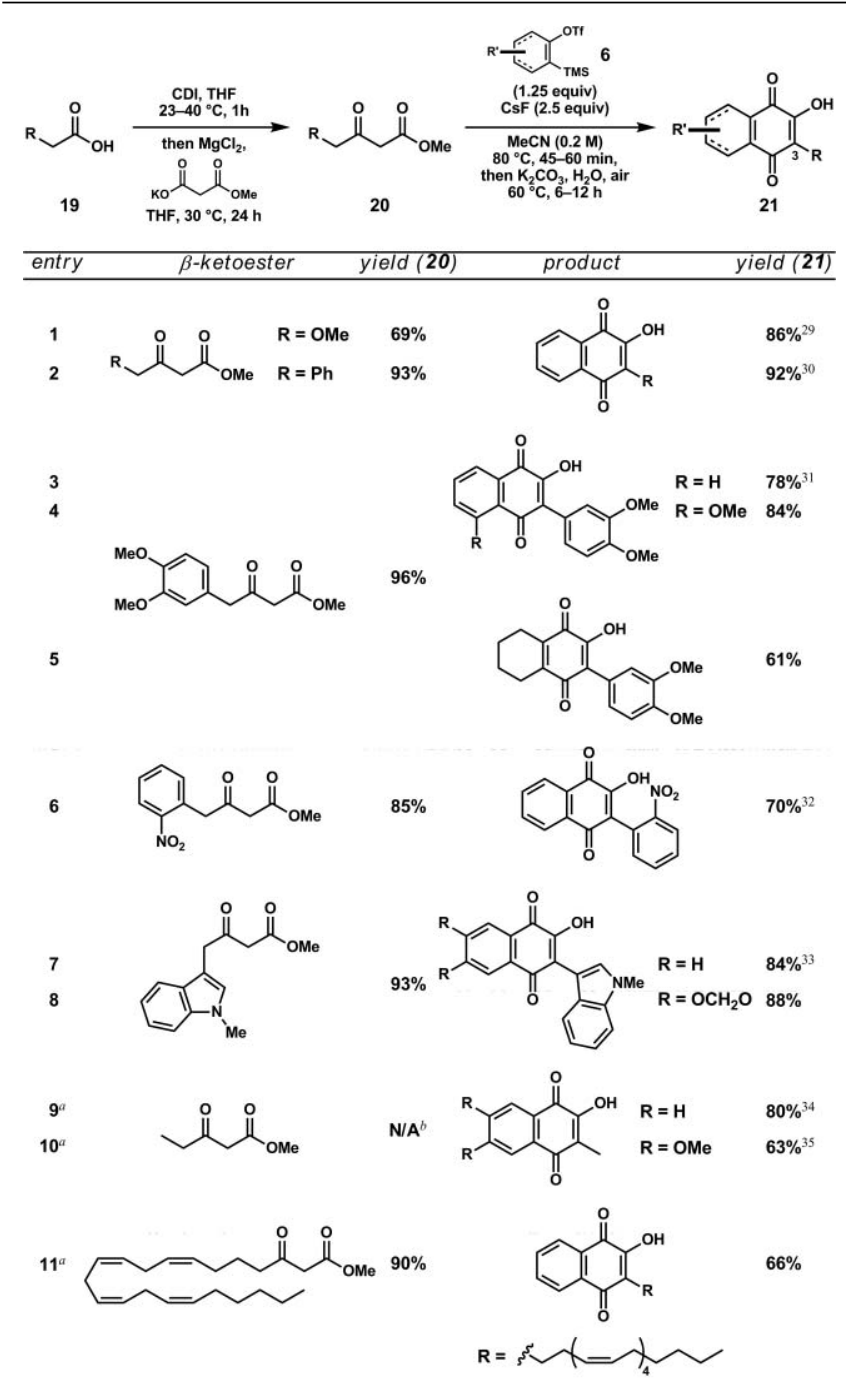

${ }^{a} \mathrm{NaOMe}$ (5 equiv) in $\mathrm{MeOH}(1.0 \mathrm{M})$ was used in place of $\mathrm{K}_{2} \mathrm{CO}_{3}$ in $\mathrm{H}_{2} \mathrm{O}$.

${ }^{b}$ Methyl 3-oxovalerate is commercially available.

In summary, we have developed an extremely concise procedure for the synthesis of 3-hydroxyisoquinolines and 2-hydroxy1,4-naphthoquinones from $\beta$-ketoesters using a novel one-pot aryne acyl-alkylation/condensation sequence. By employing this procedure in conjunction with a one-step synthesis of $\beta$-ketoesters from carboxylic acids, we are able to build these bicyclic aromatic structures in only two steps from commercially available materials. Furthermore, this method is capable of joining a variety of differentially functionalized $\beta$-ketoester and silyl aryl triflate substrates to generate products bearing a wide range of steric and electronic substitution. The utility of this approach has been demonstrated in the synthesis of the axially chiral P,N-ligand, QUINAP. Given the proven scalability of the aryne acyl-alkylation step, ${ }^{36}$ this approach is expected to enable the preparation of multigram quantities of either of these product structures. Further investigation of this methodology, including its utilization in more complex settings, is currently underway in our laboratories and will be reported in due course.

\section{Experimental}

\section{Representative procedure for the synthesis of 3-hydroxyisoquinolines from $\beta$-ketoesters}

A flame-dried $10 \mathrm{~mL}$ Schlenk flask with a septum-covered side arm equipped with a magnetic stir bar was charged with caesium fluoride $(0.152 \mathrm{~g}, 1.00 \mathrm{mmol}, 2.5$ equiv). The flask was evacuated and back-filled with argon $(\times 2)$. Acetonitrile $(2 \mathrm{~mL})$, methyl acetoacetate (1) $(0.043 \mathrm{~mL}, 0.4 \mathrm{mmol}, 1.0$ equiv) and 2(trimethylsilyl)phenyl trifluoromethanesulfonate (2) $(0.121 \mathrm{~mL}$, $0.498 \mathrm{mmol}, 1.25$ equiv) were sequentially added. The screw valve was sealed and the reaction was heated to $80{ }^{\circ} \mathrm{C}$ while stirring for $1 \mathrm{~h}$. The reaction was cooled to room temperature when TLC analysis showed complete consumption of methyl acetoacetate (1). The screw valve was removed under positive argon pressure and aqueous ammonium hydroxide $(28 \% \mathrm{w} / \mathrm{w}, 2 \mathrm{~mL})$ was added via syringe. The screw valve was replaced and the reaction was heated to $60{ }^{\circ} \mathrm{C}$ while stirring for $8 \mathrm{~h}$. The reaction was cooled to room temperature when TLC analysis showed complete consumption of the intermediate acyl-alkylated arene. The mixture was diluted with brine $(5 \mathrm{~mL})$ and extracted with EtOAc $(2 \times 15 \mathrm{~mL})$. The aqueous layer was neutralized to $\mathrm{pH} 7$ with $2.0 \mathrm{~N} \mathrm{HCl}$ and extracted again with EtOAc $(2 \times 15 \mathrm{~mL})$. The aqueous layers were discarded and the combined organic layers were extracted with $2.0 \mathrm{~N} \mathrm{HCl}(3 \times 20 \mathrm{~mL})$. The organic layers were discarded and the combined aqueous layers were neutralized to $\mathrm{pH} 7$ with $2.0 \mathrm{~N} \mathrm{NaOH}$ and extracted with EtOAc $(3 \times 20 \mathrm{~mL})$. The combined organic layers were dried over $\mathrm{MgSO}_{4}$, filtered, and concentrated under reduced pressure. The residue was purified via recrystallization from boiling EtOAc to yield 1-methyl-3hydroxyisoquinoline (4) as a yellow solid $(0.0533 \mathrm{~g}, 84 \%$ yield $){ }^{37}$

\section{Representative procedure for the synthesis of 2-hydroxy-1,4-naphthoquinones from $\beta$-ketoesters}

A flame-dried $15 \mathrm{~mL}$ reaction tube equipped with a magnetic stir bar was charged with caesium fluoride $(0.152 \mathrm{~g}, 1.00 \mathrm{mmol}$, 2.5 equiv). The reaction tube was sealed with a rubber septum, evacuated, and back-filled with argon $(\times 2)$. Acetonitrile ( $2 \mathrm{~mL}$ ), methyl 3-oxo-4-phenylbutanoate (Table 3, entry 2) (0.077 g, $0.4 \mathrm{mmol}, 1.0$ equiv) and 2-(trimethylsilyl)phenyl trifluoromethanesulfonate (2) $(0.121 \mathrm{~mL}, 0.498 \mathrm{mmol}, 1.25$ equiv) were sequentially added. The reaction was then heated to $80^{\circ} \mathrm{C}$ while stirring for $1 \mathrm{~h}$. The reaction was cooled to room temperature when TLC analysis showed complete consumption of methyl 3oxo-4-phenylbutanoate. Potassium carbonate ( 0.276 g, 5.0 equiv) in water $(2 \mathrm{~mL})$ was added via syringe and the biphasic mixture was vigorously stirred at room temperature for $30 \mathrm{~min}$. The septum was then removed and the reaction was heated to $60{ }^{\circ} \mathrm{C}$ while open to air for $12 \mathrm{~h}$. The reaction was cooled to room temperature when TLC analysis showed complete consumption of the acylalkylated arene intermediate. The reaction was diluted with EtOAc $(10 \mathrm{~mL})$ and extracted with $1.0 \mathrm{~N} \mathrm{~K}_{2} \mathrm{CO}_{3}(5 \times 15 \mathrm{~mL})$. The organic layer was discarded. The combined aqueous layers were acidified to $\mathrm{pH} 1$ with $2.0 \mathrm{~N} \mathrm{HCl}$ and extracted with EtOAc $(3 \times$ $40 \mathrm{~mL}$ ). The combined organic layers were washed with brine $(50 \mathrm{~mL})$ and dried over $\mathrm{MgSO}_{4}$. After filtration, the solvent was removed under reduced pressure and the residue was purified via flash chromatography $\left(\mathrm{SiO}_{2}, 10: 90 \mathrm{EtOAc} /\right.$ hexanes$)$ to yield 
2-hydroxy-3-phenyl-1,4-naphthoquinone as a yellow solid $(0.0918 \mathrm{~g}, 92 \%$ yield $){ }^{37}$

\section{Acknowledgements}

The authors gratefully acknowledge Pamela M. Tadross and Scott C. Virgil for helpful discussions and experimental assistance. The authors thank Abbott, Amgen, Boehringer-Ingelheim, BristolMyers Squibb, Merck, Sigma-Aldrich and Caltech for generous funding.

\section{References}

1 (a) The Way of Synthesis, ed. T. Hudlicky, and J. W. Reed, Wiley-VCH, Weinheim, 2007; (b) H. Bienaymé, C. Hulme, G. Oddon and P. Schmitt, Chem.-Eur. J., 2000, 6, 3321-3329; (c) Process Chemistry in the Pharmaceutical Industry, ed. K. G. Gadamasetti, Marcel Dekker, New York, 1999.

2 (a) Vitamins and Hormones, ed. G. Litwack, Academic Press, London, 2008, vol. 78.; (b) R. P. Verma, Anti-Cancer Agents Med. Chem., 2006, 6, 489-499; (c) M. Alvarez, and J. A. Joule, in Science of Synthesis, ed. D. S. Black, Thieme, Stuttgart, 2005, vol. 15, pp. 839-906; (d) R. M. Kanojia, O. W. Lever, Jr., J. B. Press, L. Williams, H. M. Werblood, E. C. Giardino, R. Falotico and A. J. Tobia, J. Med. Chem., 1989, 32, 990-997; (e) R. M. Kanojia, J. B. Press, O. W. Lever, Jr., L. Williams, J. J. McNally, A. J. Tobia, R. Falotico and J. B. Moore, Jr., J. Med. Chem., 1988, 31, 1363-1368.

3 (a) A. Krasovskiy, V. Krasovskaya and P. Knochel, Angew. Chem., Int. Ed., 2006, 45, 2958-2961; (b) A. Tsuboyama, H. Iwawaki, M. Furugori, T. Mukaide, J. Kamatani, S. Igawa, T. Moriyama, S. Miura, T. Takiguchi, S. Okada, M. Hoshino and K. Ueno, J. Am. Chem. Soc., 2003, 125, 12971-12979; (c) S. C. Tucker, J. M. Brown, J. Oakes and D. Thornthwaite, Tetrahedron, 2001, 57, 2545-2554; (d) J.-M. Valk, T. D. W. Claridge, J. M. Brown, D. Hibbs and M. B. Hursthouse, Tetrahedron: Asymmetry, 1995, 6, 2597-2610; (e) T. R. Kelly, A. Garcia, F. Lang, J. J. Walsh, K. V. Bhaskar, M. R. Boyd, R. Gotz, P. A. Keller, R. Walker and G. Bringmann, Tetrahedron Lett., 1994, 35, 7621-7624; (f) K. Tamao, S. Kodama, I. Nakajima and M. Kumada, Tetrahedron, 1982, 38, 3347-3354.

4 (a) K. M. Allan and B. M. Stoltz, J. Am. Chem. Soc., 2008, 130, 1727017271; (b) C. D. Gilmore, K. M. Allan and B. M. Stoltz, J. Am. Chem. Soc., 2008, 130, 1558-1559.

5 U. K. Tambar and B. M. Stoltz, J. Am. Chem. Soc., 2005, 127, 53405341.

6 U. K. Tambar, D. C. Ebner and B. M. Stoltz, J. Am. Chem. Soc., 2006, 128, 11752-11753.

7 (a) S. Krishnan, J. T. Bagdanoff, D. C. Ebner, Y. K. Ramtohul, U. K. Tambar and B. M. Stoltz, J. Am. Chem. Soc., 2008, 130, 13745-13754; (b) S. Krishnan and B. M. Stoltz, Tetrahedron Lett., 2007, 48, 75717573; (c) Q. Liu, E. M. Ferreira and B. M. Stoltz, J. Org. Chem., 2007, 72, 7352-7358.

8 H. R. Bentley, W. Dawson and F. S. Spring, J. Chem. Soc., 1952, 17631768.

9 For the first preparation of this compound, see: B. Alpha, E. Anklam, R. Deschenaux, J.-M. Lehn and M. Pietraskiewiez, Helv. Chim. Acta, 1988, 71, 1042-1052.

10 G. Tommasi, Gazz. Chim. Ital., 1920, 50, 263.

11 For the first preparation of this compound, see: R. M. Kanojia, J. B. Press, O. W. Lever, Jr., L. Williams, J. J. McNally, A. J. Tobia, R. Falotico and J. B. Moore, Jr., J. Med. Chem., 1988, 31, 1363-1368.

12 For the first preparation of this compound, see: F. Freeman, D. K. Farquhar and R. L. Walker, J. Org. Chem., 1968, 33, 3648-3650.
13 (a) B. Iglesias, D. Peña, D. Pérez, E. Guitián and L. Castedo, Synlett, 2002, 486-488; (b) N. Atanes, S. Escudero, D. Pérez, E. Guitián and L. Castedo, Tetrahedron Lett., 1998, 39, 3039-3040.

14 (a) E. R. Biehl, A. Razzuk, M. V. Jovanovic and S. P. Khanapure, J. Org. Chem., 1986, 51, 5157-5160; (b) X. Y. Han, M. V. Jovanovic and E. R. Biehl, J. Org. Chem., 1985, 50, 1334-1337; (c) H. Y. Xin and E. R. Biehl, J. Org. Chem., 1983, 48, 4397-4399.

15 D. E. Brooks, L. D.-L. Lu and S. Masamune, Angew. Chem., Int. Ed. Engl., 1979, 18, 72-74.

16 All carboxylic acid starting materials employed in Tables 2 and 3 are commercially available.

17 For the first preparation of this compound, see: R. Nowicki and A. Fabrycy, Chem. Heterocycl. Compd., 1976, 12, 910-914.

18 (a) S. Busato, D. C. Craig, Z. M. A. Judeh and R. W. Read, Tetrahedron, 2003, 59, 461-472; (b) M. B. Andrus and B. B. V. S. Sekhar, J. Heterocycl. Chem., 2001, 38, 1265-1271; (c) G. Chelucci, A. Bacchi, D. Fabbri, A. Saba and F. Ulgheri, Tetrahedron Lett., 1999, 40, 553-556.

19 N. W. Alcock, J. M. Brown and D. I. Hulmes, Tetrahedron: Asymmetry, 1993, 4, 743-756.

20 (a) J. M. Brown, D. I. Hulmes and T. P. Layzell, J. Chem. Soc., Chem. Commun., 1993, 1673-1674; (b) E. Fernandez, K. Maeda, M. W. Hooper and J. M. Brown, Chem.-Eur. J., 2000, 6, 1840-1846.

21 J. M. Brown, D. I. Hulmes and P. J. Guiry, Tetrahedron, 1994, 50, 4493-4506.

22 X. Li, L. Kong, Y. Gao and X. Wang, Tetrahedron Lett., 2007, 48, 3915-3917.

23 (a) D. Kalyani, A. R. Dick, W. Q. Anani and M. S. Sanford, Tetrahedron, 2006, 62, 11483-11498; (b) D. Kalyani, A. R. Dick, W. Q. Anani and M. S. Sanford, Org. Lett., 2006, 8, 2523-2526; (c) A. R. Dick, K. L. Hull and M. S. Sanford, J. Am. Chem. Soc., 2004, 126, 2300-2301.

24 K. Tani, D. C. Behenna, R. M. McFadden and B. M. Stoltz, Org. Lett., 2007, 9, 2529-2531.

25 (a) T. Thaler, F. Geittner and P. Knochel, Synlett, 2007, 17, 2655-2658; (b) C. W. Lim, O. Tissot, A. Mattison, M. W. Hooper, J. M. Brown, A. R. Cowley, D. I. Hulmes and A. J. Blacker, Org. Process Res. Dev., 2003, 7, 379-384.

26 Bentley et al. made a similar observation using methyl 2-(4,5dimethoxy-2-acetylphenyl)acetate and later optimized the reaction for the formation of the hydroxynaphthoquinone in 53\% yield using aqueous sodium hydroxide. See ref. 8 .

27 R. J. Anderson and M. S. Newman, J. Biol. Chem., 1933, 101, $773-$ 779 .

28 A. M. Aguinaldo, J. A. Armstrong, J. R. Cannon, S. M. Colegate, M. F. Comber, N. G. Marchant and M. V. Sargent, Aust. J. Chem., 1996, 49, 197-198.

29 For the first preparation of this compound, see: R. G. Cooke and W. R. Owen, Aust. J. Chem., 1962, 15, 486-491.

30 For the first preparation of this compound, see: T. Zincke and A. Breuer, Justus Liebigs Ann. Chem., 1884, 226, 22-60.

31 For the first preparation of this compound, see: G. Wurm and H.-J. Gurka, Pharmazie, 1997, 10, 739-743.

32 For the first preparation of this compound, see: K. Kobayashi, T. Taki, M. Kawakita, M. Uchida, O. Morikawa and H. Konishi, Heterocycles, 1999, 51, 349-354.

33 For the first preparation of this compound, see: S. Koulouri, E. Malamidou-Xenikaki, S. Spyroudis and M. Tsanakopoulou, J. Org. Chem., 2005, 70, 8780-8784.

34 For the first preparation of this compound, see: R. J. Anderson and M. S. Newman, J. Biol. Chem., 1933, 103, 405-412.

35 For the first preparation of this compound, see: A. C. Baillie and R. H. Thomson, J. Chem. Soc. C, 1966, 2184-2186.

36 D. C. Ebner, U. K. Tambar and B. M. Stoltz, Org. Synth., 2009, 86, 161-171.

37 See ESI for detailed spectroscopic data and experimental spectra. 